\title{
PERFORMANCES OF THE HIPPARCOS DATA REDUCTION ON THE GREAT CIRCLE
}

\author{
J. KOVALEVSKY 1, C. PETERSEN 2, H. VAN DER MAREL 3, F. DONATI 4 \\ 1 Observatoire de la Côte d'Azur - CERGA - Avenue Copernic - F - 06130 Grasse \\ 2 Copenhagen University Observatory - Oestervoldgade 3 - DK-1350 Copenhagen \\ ${ }^{3}$ Delft University of Technology - Thisseweg $11-$ NL-2629 Delft \\ 4 Centro di Studi Sui Sistemi - Via Vincenzo Vela, 27 - I - 10128 Torino
}

\section{Introduction}

The procedures used to reduce the Hipparcos data in both FAST and NDAC consortia follow the so-called three step procedure described in 1976 by L. Lindegren (unpublished). It consists in a first place to treat data collected during a few hours and, since the sky scanning is only slowly drifting, deduce the abscissae of observed stars on a given mean great circle, called the reference great circle (RGC). The object of this presentation is to discuss the quality of the results obtained at this stage of the reduction. The results presented here are, we believe, representative of the 3000 or so great circle reductions that shall be made during the full data treatment.

\section{Inputs to the reduction on a great circles}

Before actually computing the great circle abscissae, several tasks have to be performed in orderto prepare the input data. Although they are not made in the same order in both consortia, they ultimately lead to the same quantitics.

In a first instance, in order to linearize the great circle equations for abscissae, it is necessary to know an approximate value of the star positions. Thie is done in two steps.

i) Using the Input Catalogue or, in a later stage, an intermediate Hipparcos catalogue, a geometric position in the RGC reference system is computed for every observed star at the mean time of observation $t$. By this, we mean the position corrected for parallax, proper motion and relativistic light deflection.

ii) For each observing time $t_{i}$, the apparent position is computed using the DE200 ephemerides and orbital elements of the satellite to compute the aberration. Additional corrections to proper motion and parallax for $t_{i}-t$ are also made. It can therefore be assumed that the correction for abscissae at times $t_{i}$ are equal to the correction at time $t$.

The transformation of the position on the sky and the positions of the star on the main grid, involve the knowledge of attitude which is derived from the star-mapper observations. The accuracy with which it is derived from the Input Catalogue is given in table 1 where $\psi$ stands for along-track component of the attitude, $\theta$ is the angle between the actual rotation axis and the pole of the RGC 
and $\phi$ determines the node of the instantaneous rotation great circle on the RGC.

These numbers include the intrinsic photon count statistics, modeling errors and the inaccuracies of star positions as provided by the Input Catalogue. The later are estimated to be of the order of 0.3 arcsec r.m.s., but may be quite large, possibly a few arc seconds for some stars. They will be corrected in subsequent iterations. Modeling errors may increase when only a limited number of stars can be used in some interval of time. If this coincides with erroneous star positions, numbers of table 1 may be multiplied by a factor up to 1.5 .

Table 1

\begin{tabular}{|c|c|}
\hline Angle & Range of precision during normal operations \\
\hline$\psi$ & 35 to 65 milliarcsec \\
$\theta$ & 60 to 90 milliarcsec \\
$\phi$ & 130 to 180 milliarcsec \\
\hline
\end{tabular}

The treatment of photon counts on the main grid provides, for the mean time $t_{i}$ of each observation frame $(2.13 \mathrm{~s})$, the position of the photocenter (or some well defined point in a double or multiple star system) with respect to a slit centre. Table 2 gives, as a function of the star magnitude, the precision that is currently obtained.

Table 2

\begin{tabular}{|l|ccccccc|}
\hline \multicolumn{1}{|c|}{ Magnitude } & $<6$ & $6-7$ & $7-8$ & $8-9$ & $9-10$ & $10-11$ & $>11$ \\
\hline $\begin{array}{l}\text { Mean rms for } \\
\text { position in } \\
\text { mas }\end{array}$ & 2.5 & 4.7 & 7.3 & 10.6 & 14.5 & 21 & 33 \\
\hline
\end{tabular}

The errors are essentially due to photon statistics. Additional errors equal to one or sometimes several grid steps sometimes arise when relative positions are transformed into absolute positions on the grid. This is done from the Input Catalogue star positions and computing the corresponding grid position using attitude and the field-to-grid transformation. If there is a large error in the Input Catalogue, the slit selected may be wrong by one or even more, introducing an error in the position of one or several grid steps, that is a multiple of 1.208 arcsec.

Finally, in reckoning the observed abscissae from coordinates $G$ and $H$ on the grid, one needs to know not only the attitude of the satellite, but also the exact transformation between $G$ and $H$ and the local celestial coordinates $x$ and $y$. This is the so-called grid-to-field transformation which includes also terms in colour index $c$. It is also necessary to give the value of the basic angle. Approxi mate values of these instrumental parameters are provided from previously performed calibrations.

\section{Reduction on a great circle}

This procedure has been described in several publications (Van der Marel et al., 1989 or Van Daalen and Van der Marel, 1986). Let us only state that variations of all the input parameters that are given to this reduction and can be improved are taken as unknowns. The only quantities that cannot beimproved are the attitude and field-to-grid components perpendicular to the along-track direction. This leaves us with the following quantities to be determined : 
- Corrections $\Delta p_{\mathrm{k}}$ to the instrumental parameters

- Corrections $\Delta \phi_{\mathrm{j}}$ to the along-track attitude

- Corrections $\Delta \lambda_{\mathrm{i}}$ to the geometric abscissas on the RGC

The equations giving the difference between observed and the computed abscissae are linearized with respect to all these unknown corrections.

The following evaluation is based upon about 180 great circle reductions made either by NDAC or by FAST consortia. About half of them have been treated by both and detailed comparisons have been made between the results of some of them.

\subsection{INSTRUMENTAL PARAMETERS}

The grid-to-field transformation is presently represented by a third order polynomials in $G$ and $H$ and linear terms in $c$. Analysis of the residuals show that this model is probably insufficient and better representations may be found and used in iterations.

At present, the internal consistency of grid-to-field transformation has a mean r.m.s. residual over the field of about 0.4 milliarcsec $(0.4$ mas). However the results differ significantly more from one consortium to the other. This is partially due to a different definition of the point defined in the determination of grid coordinates (NDAC uses only the first harmonic while FAST uses a weighted mean of the first and second harmonic). This difference is of no consequence on the final results. However, when comparing the results obtained with the same definition, there remains differences which reach 2 mas in some comers of the field with an r.m.s. difference of the order of 0.6 mas. Clearly, there is room for improvement and further investigations will be made.

\subsection{ALONG-TRACK ATTITUDE}

Two methods for the attitude determination on the great circle are used in the computations.

3.2.1. Geometric solutions - In this approach, one computes a value of the attitude for the mid-time of every observation frame in which at least one star has been observed. This brings anything between 4000 and 15000 attitude unknowns per great circle. No correlation or continuity condition is introduced except for the continuity in the choice of the grid step for which reference is made to the star-mapper attitude. When remaining grid step errors are removed, the internal r.m.s. error of attitude in a geometrical solution is of the order of 10 to 15 mas.

3.2.2. Smoothed solutions - The along track attitude is represented by B-splines. This reduces the number of attitude unknowns by a factor of about 20 and a continuity condition is consistently fulfilled. In comparison with the geometric solution, the internal consistency is improved and goes down to 3-5 mas. However comparisons between both consortia show an r.m.s. of the order of 4-7 mas, mostly due to an effect with a periodicidy of one sixth of the rotation period but in which other periods sometimes also appear. Whenever the Fisher test is accepted for smoothed solution, it is applied to the determination of abscissae. In FAST, this happens most of the time except when there are large gaps in the data.

\subsection{ABSCISSA DETERMINATION}

Abscissae are the only actual astronomical result. Their precision is however closely related to the precision obtained in attitude and instrumental parameters. 
The r.m.s. residuals in both attitude determination methods are usually between 10 and 15 mas. Since there are in the mean between 18 and 36 observations of a given star, the mean geometric abscissae obtained are much more precise and have an r.m.s. between 4 and 6 mas. Table 3 gives the mean standard deviations of star abscissae in function of magnitude.

\section{Table 3}

\begin{tabular}{|c|c|c|c|c|}
\hline Magnitude & $\begin{array}{c}\text { Mean r.m.s } \\
\text { geometric }\end{array}$ & $\begin{array}{c}\text { Mean r.m.s. } \\
\text { smoothed }\end{array}$ & $\begin{array}{c}\text { r.m.s. of mean } \\
\text { grid coordinates* }\end{array}$ & $\begin{array}{c}\text { Evaluation of } \\
\text { accuracy }\end{array}$ \\
\hline$<6$ & $3 . \mathrm{mas}$ & $1.8 \mathrm{mas}$ & $0.6 \mathrm{mas}$ & $5 \mathrm{mas}$ \\
6 to 7 & $3.3 \mathrm{mas}$ & $2.2 \mathrm{mas}$ & $1.0 \mathrm{mas}$ & $6 \mathrm{mas}$ \\
7 to 8 & $3.5 \mathrm{mas}$ & $2.6 \mathrm{mas}$ & $1.5 \mathrm{mas}$ & $7 \mathrm{mas}$ \\
8 to 9 & $3.8 \mathrm{mas}$ & $3.1 \mathrm{mas}$ & $2.1 \mathrm{mas}$ & 8 mas \\
9 to 10 & $4.5 \mathrm{mas}$ & $3.8 \mathrm{mas}$ & $2.8 \mathrm{mas}$ & 9 mas \\
10 to 11 & $5.4 \mathrm{mas}$ & $4.7 \mathrm{mas}$ & $4.3 \mathrm{mas}$ & 12 mas \\
\hline
\end{tabular}

* computed assuming 25 independent observations (observation frames)

\section{Further expected improvements}

The next question that arises is what is the accuracy of these measurements? What are the additional causes of errors that do not show up in the preceding results?

We have to consider the effects of the errors on the $\theta$ and $\phi$ components of the attitude as well as the errors due to inexact values of star coordinates in the Input Catalogue. They both introduce errors in computing the a priori abscissae. A single 3 arcsec error in the catalogue position result in an error on the abscissa of up to 24 mas depending on the location of the star. This error will also affect other stars through the smearing effect caused by least squares estimation. A comparison was made by NDAC, changing the Input Catalogue with an r.m.s. of 0.27 arcsec to an improved catalogue from star-mapper with an r.m.s. of 0.185 arcsec. The r.m.s. of corresponding abscissa differences was found to be of the order of 3 mas. Therefore, it seems reasonnable to assume that there exists an additional 3 to 5 mas r.m.s. error in the first colums of table 3. An estimation of the present accuracy, taking into account this effect, is given in the last column of table 3.

In the future, when improved catalogues will be obtained from successive sphere solutions and astrometric parameter determinations, these causes of error should desappear so that the accuracy will tend towards the precision. It should become closer to the only effect of photon noise as given in table 3 and stand somewhere between the present r.m.s. estimation with the smoothing procedure and the mean r.m.s. estimation from grid coordinates.

\section{References}

Van Daalen, D.T. and Van der Marel, H., 1986, Manuscripta Geodetica, 11, p. 146.

Van der Marel, H., Van Daalen, D.T. and Petersen, C., 1989, in "The HIPPARCOS Mission", M.A.C. Perryman et al. (eds.), ESA publication SP 1111, p. 93. 